\section{SP3-16 PREVALENCE OF RISK-FACTORS OF NON-COMMUNICABLE DISEASES IN RURAL POPULATION OF INDIA}

doi:10.1136/jech.2011.1429760.16

\section{SP3-18 SESACOM-COMMUNITY HEALTH SERVICE: A MULTIDISCIPLINARY EXPERIENCE}

doi:10.1136/jech.2011.1429760.18

${ }^{1} \mathrm{~A}$ Pedrosa, ${ }^{*}{ }^{2} \mathrm{C}$ Fonsêca, ${ }^{1} \mathrm{~L}$ Góes, ${ }^{1} \mathrm{~L}$ Silva, ${ }^{1} \mathrm{M}$ Castro, ${ }^{1} \mathrm{R}$ Costa, ${ }^{1} \mathrm{~A}$ Pedrosa ${ }^{1}$ Faculdade de Medicina, Universidade Federal de Alagoas, FAMED/UFAL, Maceió, Alagoas, Brazil; 'Fundação Faculdade de Medicina, Universidade de São Paulo, FFMUSP, São Paulo, São Paulo, Brazil

Introduction Major non-communicable diseases (NCDs) include cardiovascular diseases, cancers and type 2 diabetes mellitus. The important risk factors identified for NCDs are high blood pressure, high cholesterol, being overweight or obese, and tobacco use. Primary prevention of risk factors, along with their early identification and management can help delay the progress of NCDs. The present study was undertaken with the objective of profiling riskfactors for NCDs in the rural population of Uttarakhand, India.

Methods 707 participants aged over 15 years were included. Behavioural risk factor profiles were obtained by interview, followed by anthropometric measurements and biochemical assessment of all the individuals.

Results $14.8 \%$ of the study population was found to be overweight or obese $\left(B M I \geq 25 \mathrm{~kg} / \mathrm{m}^{2}\right)$ and this was twice as common in females. Using the weight hip ratio, $44.8 \%$ population was in the moderate to high risk category (male $>0.96$, female $>0.80$ ). Overall, $6.7 \%$ of he population was found to be hypertensive. $3.7 \%$ of the subjects had diabetes (random blood glucose $>200 \mathrm{mg} / \mathrm{dl}$ ). Blood cholesterol levels were $>200 \mathrm{mg} / \mathrm{dl}$ (the at risk category) in $7.4 \%$ of subjects.

Conclusions Prevalence of NCD risk factors is lower than expected in the area. However, the "at risk" population is large and requires appropriate and timely action to prevent an epidemic of NCDs.

\section{SP3-17 COMPARISON OF TRAFFIC CRASHES INFORMATION PRESENTED BY THE I.R. OF IRAN'S SIGNIFICANT INFORMATION SOURCES WITH IN-USE MODELS OF THE USA AND DIFFERENT ASIAN COUNTRIES}

doi:10.1136/jech.2011.1429760.17

H Soori, ${ }^{*}$ M Movahedi, E Ainy. Safety Promotion and Injury Prevention Research Center, Shahid Beheshti University of Medical Sciences, Tehran, Iran

Objective Traffic crashes information presented by the I.R. of Iran's significant information sources was compared with in-use models of the USA and different Asian countries.

Methods Information on traffic crashes epidemiology was investigated and collected directly in the organisations by trained experts of the study using questionnaires verified in validity and reliability. Afterwards, information collecting and traffic injury surveillance systems of some countries were explored and after being compared with current information recording systems of the I.R. of Iran qualitatively, differences and probable weaknesses were clarified.

Results Traffic Police collects five major parts of the mentioned model in the 113 and 114 Com from format. Emergency Medicine Management Center is responsible for providing some parts of the injury surveillance system's information-which are some parts of the mentioned model-in the 115 EMS mission form format. In comparison with the American traffic records model, records of the I.R. of Iran's traffic police are similar to the model in the crash and vehicle information components completely. They are also similar in all details of the roadway and driver information components except for the traffic volume and conviction history. In comparison with some Asian countries, in the core minimum data on any case of injury class, the diagnosis of injury-related disease in not based on ICD and AIS in the I.R. of Iran.

Conclusions Traffic injury information gathering system needs utilising ICDs and AISs and-like other countries in the region-a unit format and language on traffic injuries records.
Introduction The SESACOM 2010 presents as a university extension activity of public health practices, which develops programs in basic healthcare and epidemiological studies, as the practices of the SUS, serving as a training field where can play health graduates professionals and students of health and related services, in line with the Guidelines and the new National Curriculum Framework of Medical School.

Objectives Develop health activities and preventive guidelines focus on the epidemiological profile of patients seen, collaborating to assist in basic healthcare, in the actions of promotion and prevention, at people without healthcare coverage.

Methods Actions: admission and reception of patient; performance of pre-nursing consultation; medical examination with clinical diagnosis, preventive orientation, test ordering, notions of revenue, issuing the certificates and specialty referrals; schedule and control the return of regular health events; listing of shares of health education; summary record of service and application of a questionnaire on topics of interest.

Results Contributes to the teaching program of medical school to train general practitioners, with good technical-scientific training, better able to understand the epidemiological profile of a community, ready to intervene effectively in a health reality, so that favours the articulation and strengthening of existing spaces in the associations participating in the project, and joint support from other public representations and non-governmental organisations. Conclusion The SESACOM also wants to strengthen the ties of integration of the University with the Communities, from the understanding of academic excellence with social inclusion, building increasingly the true role of a university citizen.

\section{SP3-19 PREPAREDNESS FOR MALARIA PREVENTION IN RELIEF CAMPS FOR FLOOD AFFECTEES: A CROSS SECTIONAL SURVEY FROM PAKISTAN}

doi:10.1136/jech.2011.1429760.19

B Ahmed, ${ }^{*}$ K Nanji, K Ahmed. Aga Khan University, Karachi, Pakistan

Introduction The monsoon floods in Pakistan affected about 3.2 million people, 1.4 million children and 133000 pregnant women. 1.3 million people were internally displaced. Stagnant water forms a breeding ground for mosquitoes, poses a serious threat. A survey in the relief camps of (IDP's) to evaluate the malaria prevention preparedness was conducted.

Methods A cross sectional study was conducted in October 2010. Interviews were conducted with 500 individuals, recruited through multi-stage cluster sampling. The study comprised of two phases. In the 1st phase the camps were visited and a complete checklist containing the information regarding the availability of bed nets, insecticidal sprays, mosquito repellents, coils were collected. Camps and surrounding were observed for water and sanitary conditions. In the 2nd phase of the study, the administrations of camps were interviewed regarding the measure taken for malaria prevention.

Results 500 families were interviewed. Average no of children $\leq 5$ years of age per family was 4 . None of the family reported to receive any preventive intervention. Sanitary conditions were poor with open drainage system, surrounded by stagnant water. 
Accessibility to clean water was difficult. $96 \%$ individuals reported not practicing any preventive measure for malaria. Inadequate Chloroquine was available in the medical camps, arthremeter was not available. The doctors reported, visiting of 8-10 patients daily with malaria symptoms.

Conclusion Malaria is a major epidemic and public health concern particularly during flood catastrophe. Transparent policy making is required to design strategies for the preparedness of malaria in Pakistan.

\section{SP3-20 MATERNAL IRON DEFICIENCY ANAEMIA DURING LABOUR AND ITS IMPACT ON BIRTH OUTCOMES IN WESTERN IRAN}

doi:10.1136/jech.2011.1429760.20

N Esmailnasab, ${ }^{*}$ A Afkhamzadeh. Kurdistan University of Medical Sciences, Sanandaj/ Kurdistan, Iran

Introduction Iron deficiency anaemia is one of the most common blood disorders across the world. According to the WHO, more than half of pregnant women and one third of non-pregnant women of childbearing age suffer from anaemia. The present cross-sectional study aimed to determine prevalence of maternal iron deficiency anaemia during labour and its impact on birth outcomes.

Methods A sample of 600 mothers with live births who referred to the Besat hospital in Sanandaj city western Iran was randomly recruited. Blood samples were taken and measured for Maternal $\mathrm{CBC}$ and neonatal $\mathrm{CBC}$ as well as Feretin laboratory tests.

Results Prevalence of iron deficiency anaemia among mothers and their neonates was $22.7 \%$ and $5.8 \%$ respectively with a significant correlation $(p=0.001)$. The mean haemoglobin levels were 11.95 and 11.8 in mothers who received iron supplements during pregnancy and those who did not with no significant association. Moreover, no significant relationship was observed between maternal iron deficiency anaemia and birth outcomes of LBW, prematurity, preterm labour and Apgar score.

Discussion Maternal iron deficiency anaemia was not significantly associated with adverse birth outcomes of LBW, prematurity and low Apgar score in the present study. Laboratory based interventional programs are recommended to improve prenatal care for pregnant women in developing countries' surveillance system.

\section{SP3-21 LANDMINE VICTIMS IN IRAN KURDISTAN; DEMOGRAPHIC FEATURES AND ACCIDENT CHARACTERISTICS}

doi:10.1136/jech.2011.1429760.21

${ }^{1} \mathrm{~A}$ Afkhamzadeh, ${ }^{*} \mathrm{~A}$ Delpisheh, ${ }^{3} \mathrm{H}$ Mohammadzadeh. ${ }^{1}$ Kurdistan University of Medical Sciences, Sanandaj/Kurdistan, Iran; ${ }^{2}$ llam University of Medical Science, Ilam, Iran; ${ }^{3}$ Payam Nour University of Kamyaran, Kamyaran, Iran

Background Iranian civilians living in border areas are still victims of un-neutralised war mines. This cross-sectional study aimed to assess demographic characteristics and features associated with the incident in a representative group of victims.

Materials and Methods Overall, 300 civilian mine victims in Kurdistan Province western Iran during 1991-2005 through a cluster randomised sampling method were recruited. The documentary data for those who lost their lives was gathered from the archives of local police, Red Crescent and War-disabled Organisation. Other survived mine-injured victims were interviewed for data collection using a validated checklist. SPSS was used for all analyses.

Results Overall, $17.7 \%$ of victims $(n=53)$ have been killed immediately after explosion and $82.3 \%(n=247)$ were injured. Of those survived, 40\% ( $n=99)$ had an amputation surgery of lower limb in particular. Majority of victims were either farmers, shepherd mans, or children. Among risk factors investigated, victims' job and age were significantly correlated with mine accidents in Kurdistan province after adjusting for other factors including gender, education level and socioeconomic status (Adjusted OR 2.1, 95\% CI 1.1 to 3.2, $\mathrm{p}<0.01$ and AOR 1.7, 95\% CI 1.1 to $2.5, \mathrm{p}=0.04$ respectively).

Conclusions Young civilians living in border areas between Iran and Iraq with certain jobs are still affected by un-neutralised war mines despite the grate efforts made by the government. Struggling efforts are required to avoid and minimise the adverse effects of mine expulsions in border areas in west of country and in Kurdistan in particular

\section{SP3-22 CHILD INJURY PREVENTION IN VIETNAM: ACHIEVEMENTS AND CHALLENGES}

doi:10.1136/jech.2011.142976o.22

${ }^{1} \mathrm{~S}$ Boufous, ${ }^{*}{ }^{1} \mathrm{M}$ Ali, ${ }^{2} \mathrm{H}$ Nguyen, ${ }^{3} \mathrm{M}$ Stevenson, ${ }^{1} \mathrm{R}$ Ivers, ${ }^{2} \mathrm{C}$ P Viet, ${ }^{4} \mathrm{~A}$ Nguyen. ${ }^{1}$ The George Institute for Global Health, University of Sydney, Sydney, New South Wales, Australia; ${ }^{2}$ Hanoi School of Public Health, Hanoi, Vietnam; ${ }^{3}$ Department of Epidemiology and Preventive Medicine, Monash University, Mebourne, Australia; ${ }^{4}$ The United Nations Children's Fund, Hanoi, Vietnam

Introduction As with other South Asian countries, injury is becoming a leading cause of death and morbidity among children in Vietnam. In response, government and non-government agencies in Vietnam have combined efforts during the last decade to develop and implement various child injury prevention strategies and programs. This study provides an overview of the burden of child injury, reviews relevant prevention strategies, and makes recommendations for child injury prevention in Vietnam.

Methods A review of available information related to child injury prevention in Vietnam, including peer reviewed studies, policy documents and reports from various sources, was conducted using commonly utilised databases (Medline, CINAHL, Cochrane Library, etc). Relevant grey literature was also solicited during semi-structured interviews with representatives of key stakeholders, including Ministry of Health, Ministry of Labor, WHO, UNICEF, Vietnam Red Cross, Counterpart International, Hanoi School of Public Health.

Results Drowning is the leading cause of fatal injury in Vietnamese children followed by road traffic crashes, falls, poisoning, burns and animal bites. There have been notable achievements in terms of increasing awareness of injury facing children at all levels in the community and developing a sound injury prevention policy framework in a relatively short period of time. However, much needs to be done to implement necessary environmental and legislative changes, strengthen child injury surveillance and injury prevention research; and to improve access to health services.

Conclusion The insight into the experience of Vietnam could benefit other low and middle income countries with a high burden of child injury.

\section{SP3-23 RECOGNITION OF THE OCCURRENCE OF STROKE FROM AMBULANCE CALLS}

doi:10.1136/jech.2011.1429760.23

K Ohshige. ${ }^{*}$ Yokohama National University, Yokohama, Kanagawa, Japan

Introduction Intravenous administration of tissue plasminogen activator (tPA) is effective for acute ischaemic stroke. Since there is a critical time of only a few hours to start such therapy, it is important to identify patients with stroke as soon as possible. This study aimed to establish a recognition algorithm for stroke occurrence at the time of emergency calls.

Methods The data used in the study was collected from the Yokohama emergency triage system from 1 January to 30 June 2010. In 